\title{
L’alignement pédago-quoi, vous dites? \\ Un exemple de e-évaluation dynamique formative à l'enseignement postsecondaire
}

\author{
Constructive align-what, you say? \\ An example of dynamic formative e-assessment \\ in postsecondary education
}

\section{¿Alineación pedagogi-qué, dice? Un ejemplo de evaluación electrónica formativa dinámica en la educación postsecundaria}

https://doi.org/10.52358/mm.vi9.252

Edith Potvin-Rosselet, doctorante Université du Québec à Montréal, Canada potvin rosselet.edith@courrier.uqam.ca

Jérémie Bisaillon, doctorant

Université du Québec à Montréal, Canada

bisaillon.jeremie@courrier.uqam.ca

Diane Leduc, professeure titulaire

Université du Québec à Montréal, Canada

leduc.diane@uqam.ca

\section{RÉSUMÉ}

L'article présente les étapes d'un travail de conception d'une e-évaluation dynamique formative effectué dans le cadre d'un cours de troisième cycle universitaire sur les usages pédagogiques du numérique. La e-évaluation conçue porte sur le concept d'alignement 
pédagogique et a été implémentée dans un cours d'introduction aux méthodes et stratégies d'enseignement dans sa version en ligne et asynchrone. La fonctionnalité Test dans Moodle a été utilisée pour ce faire. La e-évaluation prend la forme d'un quiz comportant huit questions et leur rétroaction pour lesquelles le processus de création est présenté. Une mise à l'essai de cette e-évaluation a eu lieu dans un cours majoritairement asynchrone. Un aperçu des résultats obtenus par les apprenantes et les apprenants ayant eu accès à la e-évaluation est brièvement présenté. Pour terminer, une appréciation globale propose des critères d'évaluation d'une e-évaluation.

Mots-clés : e-évaluation, évaluation formative, évaluation dynamique, enseignement en ligne, enseignement asynchrone, Moodle, alignement pédagogique, design pédagogique

\section{ABSTRACT}

The article presents the steps of a dynamic formative e-assessment design work carried out in a postgraduate course on distance learning. The designed e-assessment dealt with the concept of pedagogical alignment and was implemented in an introduction to teaching methods and strategies course in its online and asynchronous version. The Test feature in Moodle was used for this purpose. The e-assessment takes the form of a quiz with eight questions and its feedback for which the creation process is presented. A pilot of this eassessment took place in a mostly asynchronous course. An overview of the results obtained by learners who had access to the e-assessment is briefly presented. Finally, an overall assessment of the evaluation criteria for an e-evaluation is presented.

Keywords: e-assessment, formative assessment, dynamic assessment, online teaching, asynchronous teaching, Moodle, constructive alignment, instructional design

\section{RESUMEN}

El artículo presenta los pasos de un trabajo de diseño de evaluación electrónica formativa dinámica llevado a cabo en el marco de un curso de postgrado sobre aprendizaje a distancia. La evaluación electrónica diseñada aborda el concepto de alineación pedagógica y se implementó en un curso de introducción a los métodos y estrategias de enseñanza en su versión online y asíncrona. Para ello, se utilizó la función de prueba de Moodle. La evaluación electrónica adopta la forma de un cuestionario con ocho preguntas y su respuesta, y del cual se presenta el proceso de creación. Una prueba de esta evaluación electrónica se llevó a cabo en un curso mayormente asíncrono. Se presenta brevemente un resumen de los resultados obtenidos por los alumnos que tuvieron acceso a la evaluación electrónica. Por último, se realiza una valoración global de los criterios de evaluación para una evaluación electrónica.

Palabras clave: Evaluación electrónica, evaluación formativa, evaluación dinámica, enseñanza en línea, enseñanza asíncrona, Moodle, alineación educativa, diseño pedagógico 


\section{Introduction}

L'évaluation en contexte numérique, ou la e-évaluation, peut représenter un défi technique, notamment pour son opérationnalisation en mode asynchrone. Pourtant, plusieurs fonctionnalités sont disponibles et permettent de concevoir des évaluations de natures variées. À même un environnement numérique d'apprentissage, la mise en œuvre de ces fonctionnalités nécessite une bonne connaissance technique des outils numériques. Cependant, un savoir-faire technique ne suffit pas; une analyse et une planification adéquate des besoins d'apprentissage doivent être faites en amont. Pour ce faire, un modèle de design pédagogique est utile pour planifier la démarche menant à la réalisation d'une évaluation cohérente, puisque l'évaluation est partie prenante du cours et se veut intégrée à l'enseignement et à l'apprentissage (Biggs, 2014; Roegiers, 2000). Parmi les fonctions de l'évaluation, l'évaluation formative est reconnue pour apporter un soutien en cours de processus et son importance pour la progression des apprentissages est bien documentée (Hattie et Timperley, 2007; Leroux, 2014). L'évaluation formative peut prendre place de manière informelle, par exemple en classe lors de discussions, ou de manière plus formelle par l'entremise d'un outil spécifique d'évaluation qui demande une ou des tâches précises aux apprenantes et aux apprenants, comme un quiz, une présentation orale ou un jeu de rôle. Pour qu'elle soit efficace, l'évaluation formative doit offrir une rétroaction rapide et détaillée. Ce type de rétroaction immédiate est dite dynamique (Wang, 2010), puisqu'elle est disponible dans un court laps de temps après que l'apprenante ou l'apprenant ait exécuté la tâche demandée et que donc, contrairement à une rétroaction qui serait offerte uniquement après la tâche, la rétroaction dynamique apporte du soutien pendant la tâche. De cette manière, l'évaluation dynamique favorise la métacognition et l'autorégulation de l'apprenante et de l'apprenant (Leung, 2007). Dans un cours en ligne et asynchrone, il est important de choisir une fonctionnalité qui peut offrir une rétroaction rapide, accessible et efficace. Mettre en place une évaluation formative de qualité nécessite donc de réfléchir globalement au cours et à ses objectifs d'apprentissage, mais nécessite aussi une planification minutieuse de son contenu.

Dans le cadre du travail étudiant présenté dans cet article, une e-évaluation formative dynamique a été planifiée et mise à l'essai en contexte réel d'enseignement. Pour y arriver, un travail inspiré des étapes du modèle de design pédagogique ADDIE (Branch, 2009) a été effectué. Ce modèle comprend les étapes d'analyse (Analyze A), de design (Design D), de développement (Develop D), d'implémentation (Implement I) et d'évaluation (Evaluate E). Ces étapes ont permis d'abord d'identifier une lacune dans la séquence d'apprentissage portant sur le concept d'alignement pédagogique dans un cours de deuxième cycle universitaire enseignée par l'auteure principale de cet article (A), puis de concevoir (D et $D)$, de faire la mise à l'essai (I) et d'évaluer (E) pour apprécier la mise en place des ressources visant à combler cette lacune. La figure 1 illustre la démarche de design pédagogique du modèle ADDIE tel que proposé par Branch (2009). 


\section{Figure 1}

Le concept ADDIE

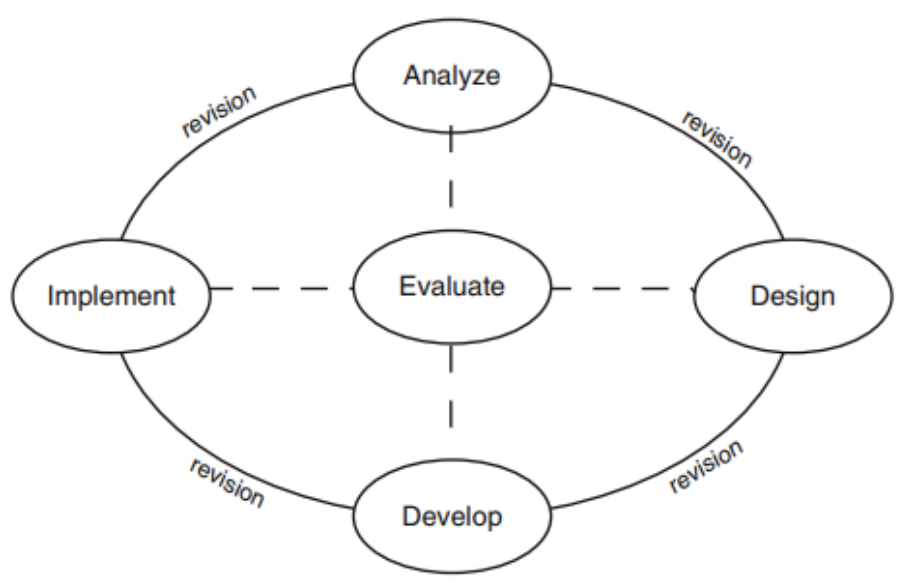

Note. Source : Branch, 2009, p. 2

Le présent article expose l'évaluation formative d'une séquence d'un cours en ligne majoritairement asynchrone. II présente tout d'abord le contexte du travail étudiant auquel la e-évaluation répond, ainsi que son principal objectif. Ensuite, cet article présente les étapes suivies pour la conception des questions formatives et de leur rétroaction (étapes $A, D$ et $D$ ). Puis, un aperçu de l'implémentation dans la fonctionnalité Test de Moodle ainsi que de la mise à l'essai en contexte réel d'enseignement est donné (étape I). Finalement, l'article met de l'avant des observations générales issues d'une réflexion autour de quelques critères d'évaluation quant à la conception de la e-évaluation (étape E). Étant donné la nature du travail étudiant effectué, il est à noter que l'utilité pédagogique de la e-évaluation dynamique est vue sous l'angle de sa conception, d'où l'utilisation d'un modèle de design pédagogique pour en faire état, et que son efficacité pour l'apprentissage reste à être formellement testée.

\section{L'objectif du travail étudiant}

Le travail de conception et de mise à l'essai d'une e-évaluation présenté dans cet article a été réalisé en 2020 dans le cadre du programme court de troisième cycle sur les usages pédagogiques du numérique (PCUPN) de I'Université du Québec à Montréal (UQAM) auquel était inscrite l'auteure principale de cet article. Lors du troisième et dernier cours de ce programme, Soutenir les apprentissages en formation à distance, il était demandé de concevoir une e-évaluation pour un contexte réel d'enseignement au postsecondaire, de choisir un outil d'implémentation adéquat pour cette e-évaluation et de justifier les choix pédagogiques liés à sa conception et à son implémentation. Ultimement, la e-évaluation pouvait être mise à l'essai dans un contexte réel d'enseignement. C'est le cours Modèles et stratégies d'enseignement au postsecondaire, un cours de deuxième cycle en formation initiale à l'enseignement, qui a été choisi pour ce faire, puisque celui-ci était, parallèlement à la conception de la e-évaluation, en transformation pour devenir un cours en ligne majoritairement asynchrone, donc contenant des activités scénarisées à même l'environnement numérique d'apprentissage Moodle. L'auteure principale était partie prenante de cette transformation, qui inclut aussi le passage vers le mode d'évaluation succès-échec, et enseignante de ce 
cours. Le contexte de cette refonte était donc propice à l'intégration de nouveaux outils d'évaluation et de nouvelles fonctionnalités numériques d'évaluation, vu son nouveau format en ligne. Ce double rôle joué dans le cours Modèles et stratégies d'enseignement au postsecondaire a été favorable à l'analyse du contexte de ce cours, des objectifs d'apprentissage et des ressources nécessaires à mobiliser pour que les apprenantes et les apprenants puissent atteindre les objectifs. La combinaison de ce contexte et de ce rôle était favorable à la réalisation du travail dont il est question dans cet article, soit de concevoir et d'implémenter une e-évaluation formative dynamique dans un contexte d'enseignement postsecondaire.

\section{Analyse du contexte}

\section{Une lacune à combler}

Le cours Modèles et stratégies d'enseignement au postsecondaire a été suivi à distance et de manière asynchrone par les cohortes de l'automne 2020 et de l'hiver 2021. Le cours consiste en quatre modules comprenant des activités d'apprentissage scénarisées à réaliser par l'entremise de Moodle (pour les activités individuelles) et de Microsoft Teams (pour les activités collectives). Dans la préparation du cours en vue de sa mise en ligne, un questionnement sur la place de l'évaluation formative a émergé, puisque la simple transposition à distance des moments de l'évaluation formative normalement réalisés en classe, souvent informels et à l'oral, poserait des défis supplémentaires dans le contexte d'activités asynchrones. En effet, les moments d'évaluation formative qui surviennent en classe lors de discussions en grand groupe, par exemple, ne peuvent avoir lieu dans cette forme dans un cours asynchrone si cela n'est pas formellement planifié à même le scénario pédagogique. C'est pourquoi les moments consacrés à l'évaluation doivent être réfléchis en cohérence avec les activités d'enseignement-apprentissage. Étant donné l'importance de la rétroaction en cours d'apprentissage (Rodet, 2000), bien planifier l'évaluation formative lors de la refonte du cours était nécessaire. Une analyse de l'alignement pédagogique du cours, soit l'analyse de la cohérence entre les objectifs d'apprentissage, les activités d'enseignementapprentissage ainsi que les activités d'évaluation (Biggs, 1996), a permis d'identifier une lacune pour ce qui est de l'évaluation formative dans la deuxième séquence d'apprentissage, soit celle portant justement sur le concept d'alignement pédagogique. Cette lacune se manifeste par l'absence d'une rétroaction rapide et spécifique sur la connaissance et la compréhension des apprenants et des apprenantes des éléments théoriques à la base du concept d'alignement pédagogique. Cette absence de rétroaction à cet endroit spécifique du cours pose problème dans la mesure où l'alignement pédagogique est un concept central des apprentissages attendus dans ce cours. Évalué de manière sommative dans toutes les productions attendues des personnes apprenantes, ce concept se doit d'être appliqué dans la production d'un plan de cours, puis réinvesti et argumenté dans la préparation et la présentation d'une leçon complète. La prochaine section décrit plus spécifiquement l'objectif d'apprentissage qu'une rétroaction à ce sujet permettrait aux apprenantes et aux apprenants d'atteindre. C'est pour pallier cette lacune qu'une e-évaluation formative et dynamique a été conçue et implémentée dans le scénario du cours Modèles et stratégies d'enseignement au postsecondaire.

\section{L'objectif de la e-évaluation}

Modèles et stratégies d'enseignement au postsecondaire est un cours d'introduction à la formation en enseignement postsecondaire; les apprenantes et les apprenants n'ont au préalable que très peu ou aucune connaissance théorique antérieure sur les théories et fondements en éducation. C'est donc leur premier contact, il faut le prétendre, avec le principe de l'alignement pédagogique tel que proposé par 
Biggs (1996). Cependant, pour atteindre l'objectif planifier une démarche cohérente d'enseignementapprentissage-évaluation, qui est l'un des objectifs d'apprentissage du cours, ils doivent être en mesure d'appliquer le principe de l'alignement pédagogique dans une situation particulière, comme la préparation d'un plan de cours. Les verbes planifier et relier contenus dans l'objectif d'apprentissage appartiennent au niveau taxonomique " appliquer », c'est-à-dire au troisième niveau de la taxonomie de Bloom révisée par Krathwol et Anderson (2009). Une tâche de ce niveau implique l'utilisation d'informations dans de nouvelles situations. Pour y arriver, et pour mettre en place une progression logique de la complexité des tâches demandées aux apprenants et aux apprenantes, une appropriation théorique de ce principe est nécessaire, soit de connaître et de comprendre les concepts principaux au cœur du principe d'alignement pédagogique. Connaître correspond à une tâche du premier niveau de la taxonomie de Bloom, et comprendre correspond à une tâche du deuxième niveau. L'évaluation formative doit donc vérifier des tâches de deux premiers niveaux du domaine cognitif en mettant en action les personnes apprenantes dans ce même type de tâches. Comme la consigne du travail étudiant à réaliser le stipulait, « [...] la e-évaluation doit permettre d'évaluer la maitrise des concepts présents dans le texte [...] " (A. Kozanitis, communication personnelle, 2019). Dans le cas présent, c'est bien le contenu de plusieurs documents portant sur l'alignement pédagogique et sur la taxonomie de Bloom qui ont été soumis à vérification par l'entremise de la e-évaluation, et non uniquement un seul texte. L'évaluation à concevoir dans le cadre du travail devait aussi « [...] permettre à l'apprenant de recevoir de la rétroaction sur la justesse ou la qualité de ses réponses » (A. Kozanitis, communication personnelle, 2019).

Plus précisément, la e-évaluation formative a comme objectif de vérifier la mémorisation (connaître) et la compréhension (comprendre) des concepts principaux de l'alignement pédagogique et de la taxonomie de Bloom. Cette e-évaluation prend la forme d'un quiz dans la page Moodle du cours Modèles et stratégies d'enseignement au postsecondaire et offre de la rétroaction aux apprenantes et apprenants après chaque question, à la manière d'une évaluation dynamique (Lepage et al., 2019).

\section{Design et développement de la e-évaluation}

\section{Création des questions}

Comme mentionné précédemment, l'analyse du contenu du cours a permis l'identification d'une lacune à combler dans la deuxième séquence du cours, soit le manque d'un dispositif d'évaluation formative du concept d'alignement pédagogique dans le scénario du cours asynchrone. La consigne du travail auquel la e-évaluation devait répondre indiquait de créer une évaluation qui vérifierait les connaissances issues de textes à lire. Avant sa transformation pour le format en ligne, les activités d'apprentissage de la deuxième séquence du cours proposaient la lecture de trois documents portant sur le concept d'alignement pédagogique, soit un diaporama sur le sujet, le blogue de Gérard (2005) et un texte introductif sur le site Internet de John Biggs (https://www.johnbiggs.com.au/academic/constructive-alignment/). C'est en partie à partir de ces documents qu'ont été élaborées les questions qui composent la e-évaluation. En effet, l'analyse du cours a permis de constater que les ressources offertes devaient être bonifiées pour permettre de mieux connaître et de mieux comprendre les éléments de base du concept d'alignement pédagogique en vue de mieux pouvoir appliquer ce concept. Ainsi, de nouvelles ressources ont été intégrées au scénario pédagogique du cours asynchrone. Ces ressources portent sur l'alignement pédagogique, mais aussi sur la taxonomie des verbes d'action de Bloom, puisque le succès de la mise en place d'un alignement pédagogique repose entre autres sur des objectifs d'apprentissage clairement exprimés, réalistes et atteignables (Leroux, 2014). Il est donc important d'apprendre à rédiger des objectifs adéquats 
et la taxonomie de Bloom est un outil indispensable pour ce faire. Le tableau 1 présente la documentation de laquelle provient le contenu à être vérifié par l'évaluation formative, c'est-à-dire les textes mis à la disposition des apprenantes et des apprenants en amont de la e-évaluation.

La forme finale de la e-évaluation formative est un quiz de huit questions dont le titre est L'alignement pédago-quoi, vous dites? Les personnes apprenantes sont amenées à faire le quiz, accessible par un lien URL à même le scénario des activités, après avoir pris connaissance de la documentation sur l'alignement pédagogique et sur la taxonomie de Bloom. Cette prise de connaissance se fait de manière progressive au cours du scénario des activités de la deuxième séquence du cours.

\section{Tableau 1}

Documentation utilisée pour appuyer le contenu théorique des questions

\begin{tabular}{|c|c|c|c|}
\hline Référence du document & $\begin{array}{l}\text { Type de } \\
\text { document }\end{array}$ & Thème principal & $\begin{array}{l}\text { Statut } \\
\text { dans le } \\
\text { cours }\end{array}$ \\
\hline $\begin{array}{l}\text { Anderson, L. W., Krathwohl, D. R., \& } \\
\text { Bloom, B. S. (2001). A taxonomy for } \\
\text { learning, teaching, and assessing: A } \\
\text { revision of Bloom's taxonomy of } \\
\text { educational objectives: Allyn \& Bacon. }\end{array}$ & $\begin{array}{l}\text { Texte - } \\
\text { article }\end{array}$ & $\begin{array}{l}\text { Taxonomie des objectifs } \\
\text { d'apprentissage }\end{array}$ & Ajouté \\
\hline $\begin{array}{l}\text { Biggs, J. (1996). Enhancing teaching } \\
\text { through constructive alignment. Higher } \\
\text { education, 32(3), 347-364. }\end{array}$ & $\begin{array}{l}\text { Texte - } \\
\text { article }\end{array}$ & Alignement pédagogique & Déjà présent \\
\hline $\begin{array}{l}\text { Biggs, J. (2014). Constructive alignment in } \\
\text { university teaching. HERDSA News, 36(3), } \\
5 .\end{array}$ & $\begin{array}{l}\text { Texte - } \\
\text { article }\end{array}$ & Alignement pédagogique & Déjà présent \\
\hline $\begin{array}{l}\text { Biggs, J. (1999). What the student does: } \\
\text { Teaching for enhanced learning. Higher } \\
\text { education research \& development, 18(1), } \\
\text { 57-75. }\end{array}$ & $\begin{array}{l}\text { Texte - } \\
\text { article }\end{array}$ & Alignement pédagogique & Ajouté \\
\hline $\begin{array}{l}\text { Biggs, J. (s.d.). Constructive alignment. } \\
\text { John Biggs. } \\
\text { https://www.johnbiggs.com.au/academic/co } \\
\text { nstructive-alignment// }\end{array}$ & $\begin{array}{l}\text { Texte - site } \\
\text { Web }\end{array}$ & Alignement pédagogique & Ajouté \\
\hline $\begin{array}{l}\text { Brabrand, C. (2006). Teaching Teaching \& } \\
\text { Understanding Understanding [vidéo]. } \\
\text { https://www.youtube.com/watch?v=fpnyPO } \\
\text { Jxy1Y\&t=172s }\end{array}$ & Film & Alignement pédagogique & Déjà présent \\
\hline $\begin{array}{l}\text { Cantin, J. [équipe APO-DSTI]. (2011, } 14 \\
\text { avril). La taxonomie d'Anderson et de } \\
\text { Krathwohl [vidéo].YouTube. } \\
\text { https://www.youtube.com/watch?v=RBPJB } \\
\text { xBvPh0 }\end{array}$ & $\begin{array}{l}\text { Capsule } \\
\text { YouTube }\end{array}$ & $\begin{array}{l}\text { Taxonomie des objectifs } \\
\text { d'apprentissage }\end{array}$ & Déjà présent \\
\hline $\begin{array}{l}\text { Gérard, L. (2015, } 25 \text { août). L'alignement } \\
\text { pédagogique : un concept clef en } \\
\text { pédagogie universitaire. Coopération } \\
\text { universitaire. } \\
\text { https://cooperationuniversitaire.com/2015/0 } \\
8 / 25 / \text { lalignement-pedagogique-le-concept- } \\
\text { cle-en-pedagogie-universitaire/ }\end{array}$ & $\begin{array}{l}\text { Texte - } \\
\text { blogue }\end{array}$ & Alignement pédagogique & Ajouté \\
\hline $\begin{array}{l}\text { Jonnaert, P. (2006). Constructivisme, } \\
\text { connaissances et savoirs. Transfert, } \\
\text { Journal semestriel de la formation }\end{array}$ & $\begin{array}{l}\text { Texte - } \\
\text { article }\end{array}$ & Constructivisme & Déjà présent \\
\hline
\end{tabular}




\begin{tabular}{|c|c|c|c|}
\hline Référence du document & $\begin{array}{l}\text { Type de } \\
\text { document }\end{array}$ & Thème principal & $\begin{array}{l}\text { Statut } \\
\text { dans le } \\
\text { cours }\end{array}$ \\
\hline $\begin{array}{l}\text { pédagogique des enseignants-stagiaires } \\
\text { du secondaire, université du Luxembourg, } \\
\text { Faculté des lettres, des sciences } \\
\text { humaines, des arts et des sciences de } \\
\text { l'éducation, vol. } 3, \text { p. } 5-9 \text {. }\end{array}$ & & & \\
\hline $\begin{array}{l}\text { Lebrun, M. [LUDOVIAMAGAZINE]. (2013, } \\
4 \text { juillet). Les compétences au cœur du } \\
\text { dispositif pédagogique [vidéo]. YouTube. } \\
\text { https://www.youtube.com/watch?v=BF7E6u } \\
6 \text { Rli0 }\end{array}$ & $\begin{array}{l}\text { Capsule } \\
\text { YouTube }\end{array}$ & Alignement pédagogique & Ajouté \\
\hline $\begin{array}{l}\text { Leduc, D. (2019a). La démarche cohérente } \\
\text { d'évaluation [vidéo]. Page Moodle du cours } \\
\text { Modèles et stratégies d'enseignement au } \\
\text { postsecondaire. UQAM. }\end{array}$ & $\begin{array}{l}\text { Capsule } \\
\text { Panopto }\end{array}$ & $\begin{array}{l}\text { Évaluation \& Alignement } \\
\text { pédagogique }\end{array}$ & Déjà présent \\
\hline $\begin{array}{l}\text { Leduc, D. (2019b). L'élaboration d'un plan } \\
\text { de cours [vidéo]. Page Moodle du cours } \\
\text { Modèles et stratégies d'enseignement au } \\
\text { postsecondaire. UQAM. }\end{array}$ & $\begin{array}{l}\text { Capsule } \\
\text { Panopto }\end{array}$ & $\begin{array}{l}\text { Alignement pédagogique } \\
\& \text { Taxonomie des } \\
\text { objectifs d'apprentissage }\end{array}$ & Déjà présent \\
\hline $\begin{array}{l}\text { Leduc, D. (2019c). Rédiger des objectifs. } \\
\text { Page Moodle du cours Modèles et } \\
\text { stratégies d'enseignement au } \\
\text { postsecondaire. UQAM. }\end{array}$ & $\begin{array}{l}\text { Document } \\
\text { Word à lire } \\
\text { et remplir }\end{array}$ & $\begin{array}{l}\text { Taxonomie des objectifs } \\
\text { d'apprentissage }\end{array}$ & Déjà présent \\
\hline
\end{tabular}

\section{Choix du format des questions}

Le tableau 2 présente les éléments constitutifs des huit questions construites. II présente d'abord le format de chacune d'elles et détaille les tâches demandées, le niveau cognitif visé par la question ainsi qu'un des deux thèmes de l'évaluation, soit l'alignement pédagogique ou la taxonomie de Bloom. Ces huit questions couvrent l'ensemble des éléments clés à évaluer pour attester d'une connaissance et d'une compréhension satisfaisantes pour les besoins du cours. Implémentées dans la fonctionnalité Test de Moodle, les questions peuvent apparaître dans un ordre aléatoire, ce qui pourrait permettre aux apprenants et aux apprenantes de reprendre le quiz à multiples reprises en amoindrissant l'effet de répétition. De plus, une rétroaction écrite pour chacune des questions a été préparée en mettant en évidence soit la réponse attendue à la question, soit une explication de la réponse attendue comme pour les questions au format à court développement. Chaque rétroaction dirige aussi l'apprenante ou l'apprenant vers des ressources supplémentaires en lien avec le contenu de la question. La prochaine section étaye le choix de cette fonctionnalité. L'annexe $B$ jointe à cet article contient un exemple du détail d'une des questions du quiz: le libellé, la rétroaction prévue ainsi que les références utilisées, le cas échéant, pour rédiger la question et la rétroaction. 


\section{Tableau 2}

Format des huit questions

\begin{tabular}{|c|c|c|c|c|}
\hline $\begin{array}{l}\text { No de la } \\
\text { question }\end{array}$ & Format & $\begin{array}{c}\text { Tâche } \\
\text { demandée* }\end{array}$ & $\begin{array}{l}\text { Niveau } \\
\text { cognitif visé }\end{array}$ & Thème abordé \\
\hline 1.1 & Dictée trouée & Complétez & Connaître & Alignement pédagogique \\
\hline 1.2 & Dictée trouée & Complétez & Connaître & Alignement pédagogique \\
\hline 2 & Appariement & Associez & Connaître & Alignement pédagogique \\
\hline 3 & Court développement & Résumez & Comprendre & $\begin{array}{c}\text { Constructivisme - } \\
\text { Alignement pédagogique }\end{array}$ \\
\hline 4 & Classement & Classez & Comprendre & Alignement pédagogique \\
\hline 5.1 & $\begin{array}{l}\text { Court développement - } \\
\text { mise en situation }\end{array}$ & $\begin{array}{l}\text { Dites et } \\
\text { expliquez }\end{array}$ & $\begin{array}{l}\text { Connaître et } \\
\text { comprendre }\end{array}$ & Alignement pédagogique \\
\hline 5.2 & $\begin{array}{l}\text { Court développement - } \\
\text { mise en situation }\end{array}$ & $\begin{array}{l}\text { Dites et } \\
\text { expliquez }\end{array}$ & $\begin{array}{l}\text { Connaître et } \\
\text { comprendre }\end{array}$ & Alignement pédagogique \\
\hline $6^{* *}$ & $\begin{array}{l}\text { Réponse courte - un } \\
\text { mot (classement) }\end{array}$ & Identifiez & Comprendre & Taxonomie de Bloom \\
\hline 7 & Court développement & Expliquez & Comprendre & Alignement pédagogique \\
\hline 8.1 & Choix multiples & Choisissez & Connaître & Taxonomie de Bloom \\
\hline 8.2 & Choix multiples & Choisissez & Connaître & Taxonomie de Bloom \\
\hline 8.3 & Choix multiples & Choisissez & Connaître & Taxonomie de Bloom \\
\hline
\end{tabular}

* La tâche demandée est le verbe d'action utilisé pour formuler la consigne donnée à l'apprenante ou à l'apprenant pour l'aiguiller dans la tâche à réaliser pour répondre à la question.

** Question inspirée de notes basées sur la taxonomie de Bloom avant révision par Anderson et Krathwol (2001). La question intègre cependant des éléments de réponse en accord avec la taxonomie révisée.

La question 1 est constituée de deux dictées trouées qui servent à vérifier la mémorisation (ce qui correspond au premier niveau cognitif de la taxonomie de Bloom révisée, connaître) de concepts de base de l'alignement pédagogique. Ce format a été choisi pour que les personnes apprenantes aient un contexte écrit (les phrases trouées) et des mots dans une banque de mots comme référence connue. En effet, les phrases et les mots s'inspirent de textes qu'elles ont lus. Le format de la question, donc la tâche demandée, combiné à son contenu, permet en effet de vérifier la mémorisation de concepts. 
La question 2 est un appariement qui sert à vérifier la mémorisation de concepts de base de l'alignement pédagogique. Ce format a été choisi pour en faire une question comportant une tâche simple. Encore une fois, les phrases et les mots s'inspirent de textes lus. Le format de la question permet de vérifier la mémorisation de concepts. En effet, les apprenantes et les apprenants n'ont pas besoin de comprendre ces concepts pour apparier une expression à sa définition.

La question 3 demande un court développement et sert à vérifier la compréhension (ce qui correspond au deuxième niveau cognitif de la taxonomie de Bloom révisée, comprendre). Ce format a été choisi pour que les apprenantes et les apprenants démontrent, par écrit, ce qu'ils comprennent de la théorie constructiviste, élément important dans la mise en œuvre du principe d'alignement pédagogique. Le format de la question permet en effet de vérifier la compréhension de ce concept.

La question 4 demande de classer des énoncés dans deux catégories. Cette question sert à vérifier la compréhension quant à certaines notions qui émanent de l'alignement pédagogique. Ce format a été choisi, parce qu'il demande une tâche cognitive différente des autres, en ce sens où l'apprenante ou l'apprenant doit faire une portion d'analyse de l'énoncé avant de pouvoir le catégoriser. Si on considère que les niveaux taxonomiques sont des continuums et non des catégories exclusives, cette question se situerait au deuxième niveau cognitif de la taxonomie de Bloom révisée.

La question 5 demande de répondre par deux courts développements et sert à vérifier la mémorisation et la compréhension des éléments principaux de l'alignement pédagogique. Par le format choisi, c'est-à-dire la mise en situation et la réponse à développement, l'apprenante ou l'apprenant doit premièrement reconnaître (premier niveau cognitif) l'alignement pédagogique, puis expliquer pourquoi il s'agit d'une situation d'alignement (deuxième niveau cognitif).

La question 6 est un type de classement où on doit identifier à quel niveau cognitif de la taxonomie de Bloom se situent les tâches ou les objectifs d'apprentissage énoncés appartiennent. Elle sert à vérifier la compréhension de la taxonomie de Bloom. Le format choisi permet d'exercer, de manière répétitive par plusieurs énoncés, la capacité à distinguer les opérations cognitives qui se cachent dans la formulation d'objectifs d'apprentissage.

La question 7 est une question à court développement et sert à vérifier la compréhension de l'alignement pédagogique. Le format choisi permet aux personnes apprenantes de démonter, par écrit, ce qu'elles comprennent globalement du principe de cohérence, élément central dans la mise en œuvre du principe d'alignement pédagogique.

La question 8 regroupe trois questions à choix multiples qui servent à vérifier la mémorisation de concepts de base du fonctionnement de la taxonomie de Bloom. Ce format diversifie le format des questions précédentes, premièrement, mais amène aussi à réfléchir à des solutions alternatives possibles pour un énoncé donné.

\section{Choix de l'outil d'évaluation}

Afin d'implémenter l'évaluation formative, la fonctionnalité Test dans Moodle a été choisie pour trois principales raisons. Premièrement, Moodle était déjà l'environnement numérique d'apprentissage dans lequel est implémenté le cours à distance. Ainsi, les apprenantes et les apprenants savent naviguer dans Moodle, sont habitués à son fonctionnement et savent qu'ils devront s'approprier de nouvelles 
fonctionnalités pédagogiques y étant incluses, comme le wiki ou le forum, au fur et à mesure du scénario de leur progression dans le scénario du cours. De plus, si une apprenante ou un apprenant éprouve une difficulté en utilisant Moodle, une aide est assurée par l'université, ce qui fait de Moodle un environnement numérique d'apprentissage accessible.

Deuxièmement, la fonctionnalité Test permet de programmer un quiz qui produit une rétroaction immédiate après chaque question. De cette manière, l'apprenante ou l'apprenant peut immédiatement savoir si sa réponse est celle attendue et sinon, en connaître la raison. L'évaluation formative a été conçue pour offrir une rétroaction écrite (et potentiellement orale à l'aide d'un fichier audio déposé dans la boîte de programmation feedback) qui l'informe de sa performance, lui explique pourquoi une réponse est adéquate, pourquoi une réponse est inadéquate, et lui propose des pistes pour se documenter, au besoin.

Troisièmement, la fonctionnalité Test de Moodle permet de créer des questions de différents formats, comme des questions à court développement, à appariement, à réponse courte ou à choix multiples, ce qui permet d'opérationnaliser la e-évaluation exactement de la manière dont le format des questions a été planifié et dont les détails sont donnés à la section précédente. Enfin, cette fonctionnalité permet de recueillir facilement des statistiques sur les résultats du quiz, comme le nombre de tentatives global et par personne, le résultat obtenu à chacune des questions, soit un succès complet, partiel ou un échec, la moyenne globale obtenue pour le quiz et le temps utilisé pour réaliser le quiz à chacune des tentatives.

\section{Mise à l'essai de la e-évaluation}

Deux mises à l'essai de la e-évaluation en contexte réel d'enseignement ont eu lieu. La première, à la session d'automne 2020, a permis à un groupe de 16 apprenantes et apprenants du cours de tester leurs connaissances et leur compréhension du concept d'alignement pédagogique. Parmi ce groupe, 14 ont décidé d'utiliser la e-évaluation comme exercice formatif. La deuxième mise à l'essai a eu lieu à la session d'hiver 2021 où 13 personnes parmi un groupe de 17 ont choisi de réaliser la e-évaluation. La consigne partagée les invitant à effectuer la e-évaluation, non obligatoire, était intégrée au scénario du deuxième module du cours et proposait de répondre à un quiz de huit questions pour lesquelles une rétroaction intégrée allait être disponible pour chaque question (voir l'annexe A pour le texte complet de la consigne introductive). Durant ce quiz, les apprenantes et les apprenants pouvaient utiliser toutes les ressources à leur disposition, disposaient d'un temps illimité pour répondre aux différentes questions et, au besoin, pouvaient reprendre le quiz ultérieurement. Des 27 tentatives effectuées, 24 indiquent un succès partiel ou complet pour toutes les questions du quiz. Les trois autres tentatives indiquent qu'une question n'a pas été réussie (question 8.2, $\mathrm{n}=1$; question 8,3, $\mathrm{n}=2$ ). De plus, de ces 27 tentatives, 21 représentent un quiz réalisé dans son entièreté. Les six autres tentatives sont des quiz où une seule question n'a pas été répondue. De ces six tentatives incomplètes, toutes indiquent que la question 3 est celle qui est restée sans réponse. La question 3 est à développement court et on pouvait y répondre à l'écrit comme à l'oral par l'entremise d'un fichier audio. La tâche demandée était de résumer dans ses mots l'hypothèse théorique qui sous-tend le paradigme constructiviste. La mise à l'essai du quiz et les 27 tentatives qui en ont découlées permettent de constater que la majorité des personnes ayant eu accès au quiz ont choisi de le passer, et que la majorité des questions du quiz ont été réussies partiellement ou complètement. Cela démontre, de manière préliminaire dans le cadre du cours Modèles et stratégies d'enseignement au postsecondaire dans sa version en ligne et asynchrone, que de tester ses connaissances et sa compréhension grâce à la e-évaluation formative sur l'alignement pédagogique a été perçu comme utile par la majorité des apprenantes et des apprenants. 


\section{Évaluation de la e-évaluation}

L'objectif de cette e-évaluation formative est de vérifier la connaissance et la compréhension d'éléments de base relatifs au concept d'alignement pédagogique tel que proposé par Biggs (1996) et à la taxonomie de Bloom révisée par Anderson et Krathwol (2001), et d'accompagner les apprenants et les apprenantes durant l'exécution de tâches cognitives par l'entremise d'une rétroaction dynamique telle que présentée en introduction de cet article. Ultimement, mieux connaître et mieux comprendre ces éléments permettra aux apprenantes et aux apprenants du cours Modèles et stratégies d'enseignement au postsecondaire, soit à ces futures enseignantes et futurs enseignants, d'appliquer concrètement le principe de l'alignement pédagogique dans la préparation de leurs cours. L'atteinte de l'objectif est partielle, puisqu'un dispositif pour tester l'efficacité de la e-évaluation comme outil d'amélioration de la connaissance et de la compréhension reste à être mis en place. Cependant, il est possible de croire que la rétroaction dynamique, de par sa nature même d'accompagnement pendant la tâche demandée à l'apprenante ou à l'apprenant, a permis un accompagnement dans l'apprentissage du concept d'alignement pédagogique. Étant donné que les consignes du travail étudiant réalisé dans le cadre du cours de troisième cycle universitaire ne contenaient pas d'exigences quant à l'évaluation (étape E) de la e-évaluation à concevoir, cette étape de la démarche de design pédagogique reste à être approfondie. Cependant, comme le suggère Branch (2009) avec le modèle ADDIE, des critères d'évaluation devraient permettre d'évaluer le produit de la démarche de design pédagogique de manière itérative, soit au moins avant et après l'implémentation en contexte réel. Ainsi, quelques critères sont suggérés dans le tableau 3 afin de contribuer à la réflexion autour de l'évaluation d'une e-évaluation formative dynamique implémentée dans un cours asynchrone où les activités d'apprentissage et d'évaluation sont scénarisées.

\section{Tableau 3}

Critères suggérés d'évaluation d'une e-évaluation formative dynamique

\begin{tabular}{|c|l|}
\hline $\begin{array}{c}N^{\circ} \\
\text { critère }\end{array}$ & \multicolumn{1}{|c|}{ Énoncé du critère } \\
\hline 1 & Accessibilité et expérience d'utilisation de la e-évaluation \\
\hline 2 & $\begin{array}{l}\text { Cohérence de la e-évaluation avec le scénario pédagogique de la } \\
\text { séquence d'apprentissage }\end{array}$ \\
\hline 3 & Pertinence du format des questions \\
\hline 4 & Qualité de la rétroaction automatique fournie \\
\hline 5 & Pertinence de la fonctionnalité numérique choisie \\
\hline 6 & $\begin{array}{l}\text { Capacité à vérifier l'atteinte de l'objectif d'apprentissage pour lequel la } \\
\text { e-évaluation est conçue }\end{array}$ \\
\hline 7 & $\begin{array}{l}\text { Flexibilité dans la conception de la e-évaluation dans l'optique de l'adapter } \\
\text { au contexte d'enseignement }\end{array}$ \\
\hline
\end{tabular}


De ces critères émerge une limite dans la démarche de design pédagogique de la e-évaluation décrite dans cet article, soit l'absence de données sur la perception de la e-évaluation, par exemple sur son accessibilité, sa pertinence dans le contexte de la séquence d'apprentissage scénarisée, ou de son efficacité auprès des personnes ayant eu accès à la e-évaluation. En effet, aucun dispositif pour les sonder n'a encore été élaboré. En ce sens, il est impossible à ce jour de discuter de l'appréciation de la e-évaluation par celles et ceux pour qui elle a été conçue. Ce travail pourrait être effectué à la suite d'une demande de certification éthique pour un travail étudiant. L'évaluation globale de la e-évaluation ici présentée permet toutefois de faire état d'une utilisation adéquate, dans le contexte, de la fonctionnalité Test dans Moodle.

\section{Conclusion}

Les étapes de planification et de mise à l'essai de la e-évaluation dynamique formative nommée L'alignement pédago-quoi, vous dites? répondent aux attentes d'un travail étudiant réalisé dans le cadre d'un cours de troisième cycle universitaire et sont présentées dans cet article. Sous forme d'un quiz comportant huit questions avec une rétroaction automatique pour chacune des questions, cette e-évaluation est implémentée par l'entremise de la fonctionnalité Test de Moodle à même le scénario pédagogique d'un cours en ligne majoritairement asynchrone.

La réalisation de ce travail a été une excellente occasion de réfléchir aux moments et aux moyens consacrés à l'évaluation dans le cours Modèles et stratégies d'enseignement au postsecondaire, où les activités d'apprentissage sont scénarisées pour répondre au format en ligne asynchrone. Une lacune sur le plan de l'évaluation formative du concept d'alignement pédagogique a été identifiée et a poussé à agir concrètement. Pour pallier cette lacune, et ultimement améliorer l'apprentissage de ce concept et favoriser son application en contexte réel, une e-évaluation alignée sur l'un des objectifs d'apprentissage du cours et sur les activités d'enseignement-apprentissage déjà installées dans le cours a été conçue. L'implémentation de cette e-évaluation est en soi une solution à la lacune identifiée, c'est-à-dire l'absence d'une évaluation formative sur le concept d'alignement pédagogique. Cependant, bien qu'il soit réaliste de supposer que la e-évaluation améliore la connaissance et la compréhension des éléments théoriques de base de l'alignement pédagogique des personnes apprenantes, la prudence est de mise lorsqu'il s'agit d'affirmer que la e-évaluation permet d'améliorer l'application du principe d'alignement pédagogique. Pour y arriver, un outil d'évaluation de la e-évaluation devrait être construit pour attester du soutien apporté dans l'atteinte de l'objectif planifier une démarche cohérente d'enseignement-apprentissage-évaluation en reliant compétences, objectifs, contenus, activités d'apprentissage et évaluation. Ce travail propose sept critères que pourrait comprendre cet outil d'évaluation. Néanmoins, la mise à l'essai de la e-évaluation atteste que le quiz de huit questions qui contient une rétroaction automatique est un moyen fonctionnel de vérifier connaissances et compréhension des éléments de base relatifs au concept d'alignement pédagogique.

Sur le plan de l'amélioration des habiletés à appliquer le concept d'alignement pédagogique en contexte réel d'enseignement, la plus-value d'une telle e-évaluation dynamique formative reste à être démontrée dans le cadre d'un cours en ligne asynchrone. Toutefois, la e-évaluation peut être intégrée à la version en présentiel et en classe du cours Modèles et stratégies d'enseignement au postsecondaire afin de formaliser le moment de l'évaluation formative portant sur le concept d'alignement pédagogique. En ce sens, le quiz en ligne L'alignement pédago-quoi, vous dites? permettrait de soutenir les apprenantes et les apprenants de cours en ligne ou en présentiel. II serait alors intéressant de réfléchir davantage à la valeur ajoutée du numérique en évaluation des apprentissages dans plusieurs contextes d'enseignement. 


\section{Liste de références}

Anderson, L. W., Krathwohl, D. R., et Bloom, B. S. (2001). A taxonomy for learning, teaching, and assessing: A revision of Bloom's taxonomy of educational objectives. Allyn \& Bacon.

Biggs, J. (1996). Enhancing teaching through constructive alignment. Higher education, 32(3), 347-364.

Biggs, J. (2014). Constructive alignment in university teaching. HERDSA News, 36(3), 5.

Branch, R. M. (2009). Instructional design: The ADDIE approach. Springer Science \& Business Media.

Gérard, L. (2015, 25 août). L'alignement pédagogique, un concept clef en pédagogie universitaire. Coopération universitaire. https://cooperationuniversitaire.com/2015/08/25/lalignement-pedagogique-le-concept-cle-en-pedagogieuniversitaire/

Hattie, J., et Timperley, H. (2007). The power of feedback. Review of educational research, 77(1), 81-112.

Krathwohl, D. R., et Anderson, L. W. (2009). A taxonomy for learning, teaching, and assessing: A revision of Bloom's taxonomy of educational objectives. Longman.

Lepage, I., Leduc, D., et Stockless, A. (2019). E-évaluation dynamique et engagement cognitif en contexte grand groupe à l'université. Évaluer. Journal international de recherche en éducation et formation, 5(3), 9-33.

Leroux, J. L. (2014). Évaluer pour faire apprendre. Dans L. Ménard et L. St-Pierre (dir.), Se former à la pédagogie de l'enseignement supérieur (p. 333-354). Montréal : Association québécoise de pédagogie collégiale.

Leung, C. (2007). Dynamic Assessment: Assessment for and as Teaching? Language Assessment Quarterly, 4(3), $257-278$.

Rodet, J. (2000). La rétroaction, support d'apprentissage? Revue du conseil québécois de la formation à distance. Téluq/UQAM.

Roegiers, X. (2000). Une pédagogie de l'intégration. Bruxelles : De Boeck Université

Wang, T. H. (2010). Web-based dynamic assessment: Taking assessment as teaching and learning strategy for improving students' e-Learning effectiveness. Computers and Education, 54(4), 1157-1166. 


\section{Annexes}

\section{ANNEXE A}

\section{Consigne introductive du quiz implémenté dans la fonctionnalité Test de Moodle}

Vous êtes invités à répondre aux huit questions du quiz qui suit, une fois votre parcours dans le Module 2 L'alignement pédagogique terminé et dans lequel vous aurez exploré différentes ressources théoriques portant sur l'alignement pédagogique et les taxonomies servant l'élaboration d'objectifs d'apprentissage.

L'objectif de cette évaluation formative est de vérifier la compréhension de notions de base relatives au concept d'alignement pédagogique de Biggs et à la taxonomie de Bloom. Vous recevrez une rétroaction pour chacune de vos réponses.

L'utilité de ce quiz est de vous offrir un outil permettant de vous assurer de bien connaître et comprendre ces deux notions, ce qui vous permettra de mieux les appliquer dans la rédaction de vos futurs plans de cours.

Ce quiz contient des questions à réponses courtes (et très courtes), des appariements, des dictées trouées et des choix multiples. Les tâches qui vous seront demandées se situent aux deux premiers niveaux de la taxonomie cognitive de Bloom, soit connaître et comprendre.

Si vous avez des questions durant ou après avoir effectué le quiz, utilisez le Forum de classe pour interpeller vos pairs ou votre enseignante.

Bon quiz! 


\section{Exemple d'une question et de sa rétroaction}

\section{QUESTION 5}

(court développement)

Lisez tout d'abord les deux situations suivantes.

\section{Situation A :}

Dans ce cours d'histoire au cégep, l'objectif de l'enseignant est de développer l'esprit critique de ses étudiants. Pour ce faire, l'enseignant donne des lectures obligatoires dans des revues scientifiques. Après chaque lecture, les étudiants doivent répondre à une série de vrai ou faux pour tester leur compréhension du texte et de ses concepts clefs. À l'examen final, une série de questions à développement court portent sur les fausses nouvelles où les étudiants doivent expliquer et mettre en relation les concepts clefs lus dans les textes obligatoires.

\section{Situation $B$ :}

Dans ce cours, l'objectif de l'enseignante est de faire appliquer aux étudiants toutes les techniques requises, étape par étape, pour la fabrication d'un masque complet adapté à la scène. Pour y arriver, l'enseignante propose différents ateliers pratiques où les étudiants s'exercent à manipuler les matériaux spécifiques à chaque technique, en plus de suivre un protocole clair. Les étudiants produisent, au fur et à mesure des ateliers, un prototype de masque sur lequel ils reçoivent une rétroaction après chaque atelier. À l'examen final, l'enseignant leur demande de créer un masque complet qui sera porté par un artiste de la scène.

5.1 Dites laquelle de ces situations démontre un plus grand alignement pédagogique et expliquez brièvement pourquoi.

Situation

5.2 Dites laquelle de ces situations démontre un moins grand alignement pédagogique et dites concrètement ce qui pourrait être fait pour « réaligner » le cours.

Situation

\section{Rétroaction}

\section{1 - Situation $\underline{\mathbf{A}}$}

Cette situation démontre un alignement pédagogique, puisque les activités auxquelles prennent part 
les étudiants (c.-à-d. les ateliers pratiques) leur permettent de développer concrètement le savoir-faire pour créer un masque; les étudiants appliquent les techniques de fabrication et reçoivent de la rétroaction. Ils sont évalués par l'entremise de la création d'un masque, ce qui correspond exactement à l'objectif d'apprentissage.

\section{2 - Situation $\underline{B}$}

Pour réaligner les méthodes d'enseignement-apprentissages (c.-à-d. les activités pédagogiques) et l'évaluation avec l'objectif de départ, il y a trois solutions possibles, puisque des actions peuvent être effectuées sur trois aspects différents du cours :

a. L'objectif d'apprentissage : il peut être reformulé pour refléter ce que les étudiants font dans le cours (c.-à-d. ce qu'ils apprennent réellement à faire, soit mémoriser (surtout) et comprendre (un peu) des concepts) ou pour refléter ce que les étudiants doivent être capables de faire à l'examen (c.-à-d. expliquer et mettre en relation des concepts).

b. Méthodes d'enseignement-apprentissage, les activités pédagogiques : les activités peuvent changer de nature ou de contenu. Les étudiants pourraient faire l'analyse de fausses nouvelles et émettre leur opinion de manière formative avant d'arriver à l'examen. L'enseignant pourrait demander aux étudiants de lire des textes scientifiques et d'ensuite les commenter à la manière d'une chronique d'opinion.

c. L'évaluation : la nature des questions d'examen peut changer pour refléter ce que les étudiants ont réellement appris, c'est-à-dire mémoriser et comprendre des concepts. Des questions à choix multiples, des vrai ou faux, des appariements et des questions à réponses courtes (comme une dictée par exemple) permettraient d'évaluer véritablement ce sur quoi les étudiants se sont exercés durant la session.

***

Complément d'information : pour revoir les textes de base qui prodiguent des explications et des définitions relatives à l'alignement pédagogique, consultez :

L'article de Biggs (1996) intitulé Enhancing teaching through constructive alignment : https://link.springer.com/article/10.1007/BF00138871

L'article de Biggs (1999) intitulé What the Student Does: teaching for enhanced learning: https://www.tandfonline.com/doi/abs/10.1080/0729436990180105

La page Constructive Alignment du site de Biggs (s.d.) :

https://www.johnbiggs.com.au/academic/constructive-alignment/

Le court texte de Bruillard (s.d.) intitulé Alignement constructif (ou constructiviste, alignement pédagogique : https://www.funmooc.fr/c4x/ENSCachan/20012/asset/efSUP S0 Bruillard alignement constructif.pdf

L'entrée nommée L'alignement pédagogique : un concept clef en pédagogie universitaire sur le blogue de Gérard (2005) : https://cooperationuniversitaire.com/2015/08/25/lalignement-pedagogique-le-concept-cleen-pedagogie-universitaire/ 